\title{
Valsartan/Sacubitril for Treatment of Hfref: A Single Center's Paradigm
}

\author{
Kathleen Lisa Morris ${ }^{1}$, Edward Shmukler ${ }^{1}$, Ann Schmit ${ }^{2}$, Sunit Preet Chaudhry ${ }^{2}$, \\ Mary Norine Walsh', Ashwin Ravichandran ${ }^{2}$ \\ ${ }^{1}$ Department of Cardiology, St. Vincent Hospital and Heart Center, Indianapolis, USA \\ ${ }^{2}$ Department of Advanced Heart Failure and Transplant, St. Vincent Hospital and Heart Center, Indianapolis, USA
}

Email address:

Kathleen.Morris2@ascension.org (K. L. Morris),Ashwin.Ravichandran@ascension.org (A. Ravichandran)

\section{To cite this article:}

Kathleen Lisa Morris, Edward Shmukler, Ann Schmit, Sunit Preet Chaudhry, Mary Norine Walsh, Ashwin Ravichandran. Valsartan/Sacubitril for Treatment of Hfref: A Single Center's Paradigm. American Journal of Internal Medicine.

Vol. 6, No. 4, 2018, pp. 52-55. doi: 10.11648/j.ajim.20180604.11

Received: April 15, 2018; Accepted: June 12, 2018; Published: July 6, 2018

\begin{abstract}
PARADIGM-HF compared valsartan/sacubitril (ARNI) with enalapril in symptomatic patients with heart failure with reduced ejection fraction (HFrEF) (1). It was stopped early after the boundary for overwhelming benefit in favor of ARNI had been reached. Patients taking ARNI had decreased symptoms, risk of HF hospitalization and all-cause and cardiovascular mortality. We sought to describe our center's initial year of experience with this novel agent. A retrospective chart review was completed of all patients in our advanced HF clinic who were prescribed ARNI between August 2015 and October 2016. Outcomes data were collected through August 2017. Consistent with the Food and Drug Administration (FDA) indication, patients treated had NYHA class II, III or IV HF symptoms with LVEF of $35 \%$ or less. The majority of those prescribed ARNI were able to initiate the medication. However, a significant proportion of patients $(26.4 \%)$ had to discontinue ARNI due to a variety of reasons, most commonly symptomatic hypotension (31.0\%) and insufficient insurance coverage (31.0\%). Only $30.5 \%$ of patients successfully treated reached the maximum dose; in $85 \%$ of these patients, hypotension limited up titration of therapy. PARADIGM-HF demonstrated benefit of ARNI therapy over enalapril in patients with HFrEF and therapy was well tolerated. In our real world experience, hypotension and lack of insurance coverage limited utilization. Further experience with this therapy in a non-trial setting will inform optimal patient selection and titration strategies. Expanded insurance coverage will be crucial to allow for patient access.
\end{abstract}

Keywords: Cardiology, Heart Failure, Medications

\section{Introduction}

The goals of heart failure management are to reduce both morbidity by improving overall quality of life and functional status along with mortality. Specifically, pharmacologic therapy in $\mathrm{HFrEF}$ are to also slow or reverse the deterioration in myocardial function due to pathologic remodeling. Over the years, there have been major strides in management of HFrEF patients that have significantly improved both morbidity and mortality.

Since the results of the CONSENSUS trial showed a $16 \%$ mortality reduction in those with mild to moderate symptoms of heart failure with reduced ejection fraction (HFrEF), angiotensin-converting enzyme (ACE-I) inhibitors have been the cornerstone of treatment [2]. Further advances in medical therapy were realized when beta blockers, mineralocorticoid receptor antagonists (MRA) and fixed dose hydralazinedinitrate were found to have profound benefits in HFrEF [310]. Subsequently, cardiac resynchronization therapy (CRT) demonstrated similar benefit [11-13].

For the first time in over a dozen years, the PARADIGM$\mathrm{HF}$ trial found benefit for a new class of medication: the angiotensin receptor antagonist/neprilysin inhibitor (ARNI) valsartan/sacubitril. After a 2 week run-in phase of enalapril $10 \mathrm{mg}$ twice daily, compared to patients taking enalapril, those taking ARNI had decreased all-cause mortality, mortality due to cardiovascular causes and decreased risk of hospitalization for HF. While patients did demonstrate a reduction in HF symptoms, they also experienced more 
frequent hypotension and angioedema (not life threatening), but no worsening renal impairment, hyperkalemia or cough.

In the wake of these findings, ARNI was approved by the FDA and became commercially available in July 2015. Farmakis et al discuss the practical concerns on the initiation of therapy with the necessity of close monitoring for side effects including hypotension and hyperkalemia [14]. Kobalava et al describe their initial experience with ARNI in a small patient population showing favorable results, specifically in relation to patient dynamics of biomarkers [15]. However, overall, Information on patient tolerance, discontinuation of drug and side effect profile and patient access to therapy remains limited in a practical setting, especially when many are using this novel agent de novo, thereby nullifying the runin phase aspect of PARADIGM-HF.

We sought to describe our initial experience with efficacy, safety, tolerability and reasons for discontinuation of ARNI in patients followed in our advanced HF clinic during our first year of experience.

\section{Methods}

\section{Study Oversight}

The study protocol was approved by the St. Vincent Hospital institutional review board. Data was collected, managed and analyzed by the authors. All authors made the decision to submit the manuscript for publication and assume responsibility for the accuracy and completeness of the analysis.

\section{Study Design}

A retrospective chart review was completed of all 151 patients in our advanced HF clinic who were prescribed ARNI between August 2015 and October 2016. Patients had New York Heart Association (NYHA) class II-IV HF symptoms and LVEF $<35 \%$. Electronic records of patients were reviewed to extract the relevant data.

Primary outcomes evaluated were cardiovascular and allcause mortality, HF hospitalization, safety, tolerability and reasons for discontinuation.

\section{Results}

Baseline characteristics of the patient population are seen in table 1. One hundred-fifty-three patients met the prespecified inclusion criteria, with a mean follow-up period of 1.3 years. Compared to patients enrolled in PARADIGM-HF, patients were more frequently female, more symptomatic, and more commonly had a non-ischemic etiology of cardiomyopathy. There was also a greater use of MRA, implantable cardioverter defibrillator (ICD) and CRT. Although left ventricular ejection fraction (LVEF) was lower in our patients, baseline blood pressures wer similar to PARADIGM-HF. The majority of patients $(91.3 \%)$ were on ACE-I/ARB prior to initiation of ARNI.
Table 1. Baseline Characteristics of Advanced Heart Failure Patients.

\begin{tabular}{ll}
\hline Total Number of Patients & 151 \\
Age - yr & 59.6 \\
Female Sex - no. (\%) & $47(31 \%)$ \\
Race & \\
White & $120(80.7 \%)$ \\
Black & $30(19.8 \%)$ \\
Asian & $1(0.01 \%)$ \\
Body Mass Index & 33.5 \\
Average Baseline Blood Pressure & $120 / 74$ \\
Serum Cr & $1.04(0.6-1.8)$ \\
Ischemic Cardiomyopathy & $72(47 \%)$ \\
LV ejection fraction & $23.6(10-40)$ \\
NYHA & \\
I & $0(0.0 \%)$ \\
II & $74(47.7 \%)$ \\
III & $65(43.7 \%)$ \\
IV & $5(3.2 \%)$ \\
ICD & $116(77.5 \%)$ \\
CRT & $44(29 \%)$ \\
Beta Blocker & $144(96.7 \%)$ \\
ACE-I/ARB & $137(91.4 \%)$ \\
Mineralocorticoid antagonist & $104(70.1 \%)$ \\
Nitrates + Hydralazine & $9(5.9 \%)$ \\
Median follow up & 1.33 years \\
\hline
\end{tabular}

Approximately one quarter $(26.5 \%)$ of the patients discontinued ARNI during the study period. The most common reasons for discontinuation, as seen in Table 2, were symptomatic hypotension $(31.0 \%)$ and insurance difficulty $(31.0 \%)$. Angioedema was also seen in $7 \%$ of patients.

Table 2. Reasons for discontinuation of ARNI.

\begin{tabular}{ll}
\hline Reason & Number of patients \\
\hline Hypotension & 13 \\
Insurance & 13 \\
Death & 4 \\
Angioedema & 3 \\
Syncope & 2 \\
Hospitalization & 1 \\
VAD & 1 \\
Hives & 1 \\
Acute Kidney Injury & 1 \\
Nausea & 1 \\
Diarrhea & 1 \\
Transplant & 1 \\
Total & 42 \\
\hline
\end{tabular}

$30.5 \%$ of patients were able to tolerate the maximal dose of ARNI (Table 3), with the major limiting factor for up titration being hypotension (Table 4). Hyperkalemia did limit titration in some patients but was not a reason for discontinuation. 
Table 3. Maximally tolerated dose (of patients who started the medication).

\begin{tabular}{llll}
\hline Dose (Sacubitril/Valsartan) & $\mathbf{2 4} \mathbf{~ m g} / \mathbf{2 6} \mathbf{~ m g ~ B I D}$ & $\mathbf{4 9} \mathbf{~ m g} / \mathbf{5 1} \mathbf{~ m g ~ B I D}$ & $\mathbf{9 7} \mathbf{~ m g} / \mathbf{1 0 3} \mathbf{~ m g ~ B I D}$ \\
\hline Number of patients & $50(33.1 \%)$ & $52(34.4 \%)$ & $46(30.5 \%)$ \\
\hline
\end{tabular}

Table 4. Reasons for not reaching maximum dose.

\begin{tabular}{ll}
\hline Reason & Number of patients \\
\hline Medication Stopped & 42 \\
Hypotension & 46 \\
Hyperkalemia & 4 \\
Renal dysfunction & 1 \\
Syncope & 1 \\
Other & 2 \\
\hline
\end{tabular}

*Other includes provider discretion due to stability of regimen and patient preference.

\section{Discussion}

Among patients with HF with reduced ejection fraction, PARADIGM-HF demonstrated a clear benefit of treatment with ARNI compared to enalapril. Adoption of new therapies in a real world population can result in different tolerability and outcomes [16]. We therefore sought to describe our initial experience regarding efficacy, safety an d tolerability of ARNI therapy in a practical, non-clinical trial setting.

The baseline characteristics of our patients differed from those enrolled in PARADIGM-HF. Our patients were more symptomatic with $44 \%$ demonstrating NYHA Class III symptoms at the time of drug initiation compared to $23-25 \%$ of patients enrolled in PARADIGM-HF. This likely reflects the fact that our HF clinic tends to be a regional center for tertiary level care. While the majority of patients were on ACE-I/ARB prior to ARNI initiation, the majority. Our patient population also included a higher proportion of women and those with non-ischemic etiology of HF. Use of CRT-D, ICD and MRA was also greater in our patients, an expected finding for our practice when comparing the largely non-United States population in the PARADIGM-HF trial.

Hypotension was the most common reason for both discontinuation of therapy and inability to reach a maximum dose of ARNI, for which multiple explanations may exist. Patients enrolled in PARADIGM-HF underwent a run-in phase where they had to tolerate enalapril $10 \mathrm{mg}$ BID and ARNI 200mg BID prior to being randomized to enalapril versus ARNI. Although only $8.7 \%$ of the patients in our clinic were not on an ACE-I/ARB prior to initiating ARNI therapy, there was no minimum ACE-I/ARB dose requirement prior to initiating ARNI. In addition, our patients had worse baseline functional status and lower LVEF, which may have decreased ARNI tolerability. Finally, the greater use of MRA along with ARNI may have contributed to the intolerance, particularly hypotension and/or hyperkalemia.

Hyperkalemia serious enough to cause discontinuation or halting of ARNI titration was rare in our series. Although hospitalizations and mortality due to hyperkalemia increased following the publication of RALES [9] (showing the benefit of spironolactone in HFrEF), this did not occur in PARADIGM-HF or in our population, likely due to careful monitoring of potassium and renal function after drug initiation.

Insurance coverage limited many patients who were prescribed ARNI. Our clinic used a strategy of using sample medication at time of initiation, followed by prescription of ARNI for those patients who tolerated the therapy. In this way, prior authorization and appeals were focused on patients who were stable on ARNI. Despite aggressive efforts by our seasoned HF clinic nurses and cardiologists, financial constraints played a significant role in the discontinuation of ARNI. Some patients lacked any insurance coverage for ARNI, whereas others had significant co-pays making continued ARNI use too burdensome. Our data was collected prior to the publication of the American College of Cardiology/American Heart Association guideline update giving ARNI therapy a Class IB recommendation [17]. This recommendation and increasing experience with the new therapy may change insurance coverage going forward.

\section{Conclusion}

PARADIGM-HF clearly demonstrated benefit of ARNI over ACE-I therapy in patients with HFrEF. In our real world experience, we found that tolerability and cost limited ARNI use. Further experience with this therapy in a non-trial setting will inform optimal patient selection and titration strategies to improve overall tolerability. However, expanded and early insurance coverage will be crucial to allow for patient access to medications with significant benefit to allow early clinical improvement, particularly in diseases such as systolic heart failure that carry a high mortality.

\section{References}

[1] McMurray JJV, et al. Angiotensin-neprilysin inhibition versus enalapril in heart failure. N Engl J Med 2014; 371: 993-1004.

[2] CONSENSUS Trial Study Group. Effects of enalapril on mortality in severe congestive heart failure, results of the cooperative north Scandinavian enalapril survival study. $\mathrm{N}$ Engl J Med 1987; 316(23):1429-35.

[3] Packer M, et al. Effect of Carvedilol on the Morbidity of Patients with Severe Chronic Heart Failure. Circ 2002; 106 (17): 2194-9.

[4] Lechat P, et al. A randomized trial of beta-blockade in heart failure. The Cardiac Insufficiency Bisoprolol Study (CIBIS). Circ 1994; 90 (4): 1765-1773.

[5] Fagerberg, B, et al. Effect of metoprolol CR/XL in chronic heart failure: Metoprolol CR/XL Randomised Intervention Trial in Congestive Heart Failure. Lancet 1999; 353 (9169): 2001-7. 
[6] Poole-Wilson PA, et al. Comparison of carvedilol and metoprolol on clinical outcomes in patients with chronic heart failure in Carvedilol or Metoprolol European trial. Lancet 2003; 362 (9377): 7-13.

[7] Swedberg $\mathrm{K}$ et al Eplerenone and atrial fibrillation in mild systolic heart failure: results from the EMPHASIS-HF (Eplerenone in Mild Patients Hospitalization And Survival Study in Heart Failure) study. J Am Coll Cardiol 2012; 59(18):1598-603.

[8] Pitt B, et al. The EPHESUS trial: eplerenone in patients with heart failure due to systolic dysfunction complicating acute myocardial infarction. Eplerenone Post-AMI Heart Failure Efficacy and Survival Study. N Engl J Med 2003; 348:1309-21.

[9] Pitt B, et al. The effect of spironolactone on morbidity and mortality in patients with severe heart failure. New Engl J Med 1999; 341(10):709-17.

[10] Taylor AL, et al. Combination of Isosorbide Dinitrate and Hydralazine in Blacks with Heart Failure. New Engl J Med 2004; 351(20):2049-57.

[11] Abraham WT, et al. Cardiac Resynchronization in Chronic Heart Failure. New Engl J Med 2002; 345: 1845-53.
[12] Cleland, et al. The Effect of Cardiac Resynchronization on Morbidity and Mortality in Heart Failure. New Engl J Med 2005; 352: 1539-49.

[13] Moss AJ, et al. Cardiac-Resynchronization therapy for the prevention of heart failure events. New Engl J Med 2009; 361 (14):1329-1338.

[14] Farmakis D, et al. Practical considerations on the introduction of sacubitril/valsartan in clinical practice: Current evidence and early experience. Int J Cardiol 2016; 223: 781-84.

[15] Kobalava $\mathrm{ZhD}$, et al. First Experience of Clinical Application of LCZ696 - an AT1-angiotensin Receptors and Neprilysin Inhibitor-in Patients with Chronic Heart Failure and Reduced Ejection Fraction. Kardiologiia. 2015; 55: 14-25.

[16] Juurlink DN, et al. Rates of Hyperkalemia after Publication of the Randomized Aldactone Evaluation Study. New Engl J Med 2004; 351: 543-51.

[17] Yancy CW, et al. 2016 ACC/AHA/HFSA Focused Update on New Pharmacological Therapy for Heart. Failure: An Update of the 2013 ACCF/AHA Guideline for the Management of Heart. Failure. J Am Coll Cardiol 2016; 68 (11): 1476-88. 